\title{
LOCALIZATION AND GENUS IN GROUP THEORY
}

\author{
G. PESCHKE
}

\begin{abstract}
We provide a unifying category theoretical framework to discuss various kinds of local global phenomena. Specializing to localization of groups at sets of primes $P$, we identify a large class of groups for which localization supports a passage from local information to global information. Local global principles for groups in this class are established and used to calculate certain homomorphism sets as well as splittings of epimorphisms and monomorphisms from local data.
\end{abstract}

\section{INTRODUCTION}

Let $\mathscr{L}$ be a family of localizing functors on a category $\mathscr{C}$, as described in Adams [1]. We would then like to obtain information abut the objects and morphisms in $\mathscr{C}$ from their localizations by the functors in $\mathscr{L}$. Such methods, called local global principles, are now in common use in subjects like number theory, commutative algebra, and homotopy theory of nilpotent spaces. In addition various notions like "genus" [13], "type" [21], "clone" [14], etc., have been introduced to refer to collections of objects which have isomorphic local data.

Here we extend the method of local global principles to the category of groups, and we consider the resulting genera of groups. Our approach is guided by a general unifying category theoretical framework for the discussion of local global phenomena. The key ideas underlying this framework are outlined in A, B, C below. Details can be found in $\S 1$.

A. If $\mathscr{L}$ is a family of localizing functors on a category $\mathscr{C}$, then the members of $\mathscr{L}$ are related by natural transformations coming from the following $\mathscr{C}$ intrinsically defined partial ordering: $L_{\mu} \geq L_{\nu}$ if the class of $L_{\mu}$-local objects (objects for which the localizing map $X \rightarrow L_{\mu} X$ is an isomorphism) contains all $L_{\nu}$-local objects. We form the category $\mathscr{L} \mathscr{C}$ whose objects are diagrams in $\mathscr{E}$ modeled on the partial order of $\mathscr{L}$. In particular, applying the functors in $\mathscr{L}$ to an object $X \in \mathscr{C}$ yields an object $\mathscr{L}(X) \in \mathscr{L} \mathscr{C}$. This process is a functor $\mathscr{L}: \mathscr{C} \rightarrow \mathscr{L} \mathscr{C}$.

B. The extent to which $\mathscr{L}$-local data actually determine objects in $\mathscr{C}$ is measured by the "size" of the fibers of the functor $\mathscr{L}: \mathscr{C} \rightarrow \mathscr{L} \mathscr{C}$. Thus we define the genus of $\underline{X} \in \mathscr{L} \mathscr{C}$ to be the collection of all isomorphism classes of objects

Received by the editors November 10, 1993.

1991 Mathematics Subject Classification. Primary 18E35, 20E34, 55P60.

The author was supported by NSERC of Canada. 
$X \in \mathscr{C}$ with $\underline{X} \cong \mathscr{L}(X)$. We say that objects $X$ and $Y$ of $\mathscr{C}$ belong to the same $\mathscr{L}$-genus if $\mathscr{L}(X) \cong \mathscr{L}(Y)$.

C. If $\mathscr{C}$ has inverse limits, we define the $\mathscr{L}$-completion of $X \in \mathscr{C}$ to be $\lim \mathscr{L}(X)$. The functor $\mathscr{L}: \mathscr{C} \rightarrow \mathscr{L} \mathscr{C}$ is left adjoint to the inverse limit functor, and, in particular, there is a natural map $c: X \rightarrow \lim \mathscr{L}(X)$. If $c$ is an isomorphism, the object $X$ is determined by its $\mathscr{L}$-local data and morphisms from an object $A$ into $X$ correspond bijectively to morphisms between the local diagrams $\mathscr{L}(A) \rightarrow \mathscr{L}(X)$.

Guided by this general framework we approach the development of local global principles for $P$-localizing functors on the category of groups. To explain this, recall that, for a set of primes $P$, a group $G$ is $P$-local if $G$ has unique $q$ th roots for every $q \notin P$. Further the $P$-localization of a given group $G$ is a universal homomorphism $G \rightarrow L_{P} G$, where $L_{P} G$ is $P$-local; see $\S 0$ for precise definitions and basic properties. We consider families $\mathscr{L}$ of $P$-localizing functors which are of the form $\left\{L_{P_{1}} \rightarrow L_{Q}, L_{P_{2}} \rightarrow L_{Q}, \ldots\right\}$, where $P_{1}, P_{2}, \ldots$ are sets of primes containing $Q$ and every prime not in $Q$ belongs to exactly one of the sets $P_{\mu}$. Theorem 2.1 gives a general criterion under which the map $c: G \rightarrow \lim \mathscr{L}(G)$ is an isomorphism. In $\S 3$ we describe groups for which this criterion is easy to check. For a group $G$ in this class the map $c$ is an isomorphism if $G$ has torsion in only finitely many of the sets $P_{k}$; see Theorem 4.1. Such a result has previously only been available in the case where $G$ is a finitely generated nilpotent group; see [13].

In $\S \S 5$ and 6 we give examples for building homomorphisms between groups from local data. In $\S 5$ we deal with homomorphisms out of an infinite product of torsion groups. In $\S 6$ we show how to obtain 1-sided inverses for epimorphisms and monomorphisms from local data. In $\S 7$ we characterize the $\mathscr{L}$-genus of a given group.

To put this study somewhat into perspective, let us recall that the $P$-localization of an arbitrary group was first introduced by Ribenboim [17] in 1978. At this level of generality $P$-localization methods have, so far, not found systematic use. Probably this has to do with the following: $P$-localizing a group $G$ annihilates torsion of $G$ at all primes $q \notin P$. If $G$ is arbitrary, this can entail destroying more than just $P$-complementary torsion structure of $G$, in which case one is left with too little local information to work with. Thus it becomes necessary to determine for which groups $P$-localization is reasonably well behaved. This is done in $\S 3$, where we describe large categories of groups on which $P$-localization behaves nicely. Whether or not the $P$-localizing functor $L_{P}$ behaves nicely on $G$ depends primarily on the torsion structure of $G$. Our choice of families of localizing functors $\mathscr{L}$, as described above, is suggested by the need to accommodate various kinds of torsion structure.

We remark that the results in this paper are likely to extend to analogous statements about $P$-localizing functors in the homotopy category of $\mathrm{CW}$-complexes. For example, the problem of splitting an epimorphism of groups (treated here in $\S 6$ ), corresponds to the problem of finding a section for a fibration. Indeed, the general category theoretical principles governing local global principles, expounded in $\S 1$, apply to the homotopy category of CW-complexes as well (modulo Remark 1.9). Moreover, combining classical work and [8], we see that all remaining potential obstructions to extending local-global principles 
from groups to spaces have to do with the fundamental group and its action on higher homotopy groups. Some of these potential obstructions are adequately dealt with in this paper.

\section{Preliminaries}

Here we collect the relevant definitions and properties of $P$-localization of groups. Throughout, the symbol $P$ will denote a set of primes. We write $n \in P^{\prime}$ to denote an integer in the multiplicative closure of the set of primes which do not belong to $P$.

A group $G$ is $P$-local if, for every $n \in P^{\prime}, n$th roots exist in $G$ and are unique; i.e. if the self-map of $G, g \mapsto g^{n}$, is bijective. There exists a $P$ localizing functor $L_{P}: \mathscr{G} \rightarrow \mathscr{G}_{P}$, from the category of groups $\mathscr{G}$ to the full subcategory of $P$-local groups $\mathscr{G}_{P}$; see [17, 18,9]. One way to construct $L_{P}$ is as follows. If $g \in G$ and $n \in P^{\prime}$, introduce a new symbol $x_{g, n}$ and define the $P^{\prime}$-root creating functor to be

$$
\sqrt{ }_{P} G:=\left(G *\left\langle x_{g, n}: g \in G, n \in P^{\prime}\right\rangle\right) / g=\left(x_{g, n}\right)^{n} .
$$

By design, $G$ is a subgroup of $\sqrt{ }_{P} G$ and every $g \in G$ has $n$th roots in $\sqrt{ }_{P} G$, for each $n \in P^{\prime}$.

Let $U_{P}$ be the functor which makes $P^{\prime}$-roots unique: $U_{P} H:=H / K$, where $K$ is the intersection of all kernels of maps from $H$ to a group in which $P^{\prime}$-roots are unique. Then define

$$
L_{P} G:=\lim _{\longrightarrow}\left(G \rightarrow\left(U_{P} \sqrt{ }_{P}\right) G \rightarrow\left(U_{P} \sqrt{ }_{P}\right)^{2} G \rightarrow \cdots\right) .
$$

$L_{P} G$ is $P$-local, and the canonical map $\varepsilon_{P}: G \rightarrow L_{P} G P$-localizes. Universal properties of the operations $\sqrt{P}_{P}, U_{P}$, and $\lim _{\longrightarrow}$ show that $\varepsilon_{P}$ is a natural transformation $\mathrm{Id}_{\mathscr{G}} \rightarrow L_{P}$. Its relevant properties are collected in the theorem below.

0.1 Theorem. The pair $\left(L_{P}, \varepsilon_{P}\right)$ forms an idempotent monad with the following properties.

(i) Universal property: If $H$ is a P-local group, then every homomorphism $u: G \rightarrow H$ factors uniquely through $\varepsilon_{P}: G \rightarrow L_{P} G$.

(ii) $L_{P}$ preserves epimorphisms.

(iii) $L_{P}$ commutes with colimits.

(iv) $i \circ L_{P}$ commutes with directed colimits, where $i: \mathscr{G}_{P} \rightarrow \mathscr{G}$ is the inclusion functor.

(v) $L_{P}$ commutes with restricted products. In special situations, additional information is available:

(vi) $L_{P}$ is right exact on central extensions.

(vii) On nilpotent groups, $L_{P}$ coincides with the functor described in $[11,13]$. $L_{P}$ does not increase the order of nilpotency. Moreover, a homomorphism $\varepsilon_{P}: G \rightarrow H \quad P$-localizes if and only if $H$ is $P$-local, $\operatorname{ker}\left(\varepsilon_{P}\right)$ consists of all $P^{\prime}$-torsion elements of $G$, and, for every $h \in H$, there exists an integer $n \in P^{\prime}$ such that $h^{n} \in \operatorname{im}\left(\varepsilon_{P}\right)$.

Proof. (i) This follows from the universal property of free products and kernels.

(ii) This is true because the operations $\sqrt{ }_{P}$ and $U_{P}$ both preserve epimorphisms. 
(iii) This is true because $L_{P}$ is left adjoint to the inclusion $\mathscr{G}_{P} \hookrightarrow \mathscr{G}$; a reformulation of (i).

(iv) If $\bar{G}$ is a directed system of groups, $P$-localization yields the directed system of $P$-local groups $\overline{L_{P} G}$. Its colimit is a $P$-local group $\Gamma$, as can be shown by using the description of a directed colimit of groups in [20].

There is a canonical map $e: \lim \bar{G} \rightarrow \Gamma$ determined by the $P$-localizing maps $\varepsilon_{\lambda}: G_{\lambda} \rightarrow L_{P} G_{\lambda}$. To see that $e \quad P$-localizes we check that it has the universal property 0.1 (i). Let $H$ be a $P$-local group. If $f: \lim \bar{G} \rightarrow H$ is given, then the universal property of the $P$-localizing maps $\varepsilon_{\lambda}$ yields uniquely the maps $f_{\lambda}$ making the part of the diagram below commute which does not involve $\Gamma$ : The maps $f_{\lambda}$ form a cone $\overline{L_{P} G} \rightarrow H$. So the universal property of the colimit $\Gamma$ yields uniquely the arrow $\varphi$ which can be seen to make the whole diagram commute.

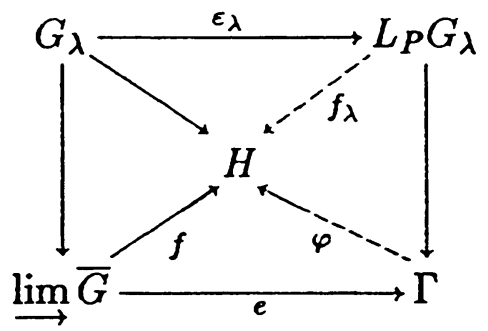

(v) $L_{P}$ commutes with finite products and, by (iv), with restricted products; compare [19, Proposition 6.6].

(vi) A central extension $A \stackrel{i}{\rightarrow} G \stackrel{v}{\rightarrow} R$ yields the commutative diagram

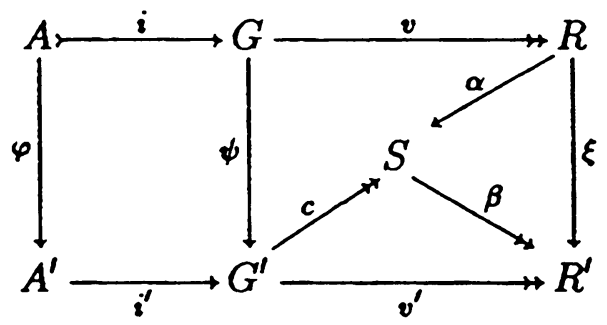

in which a group $H^{\prime}$ is defined as $H^{\prime}:=U_{P} \sqrt{P}_{P} H$. By induction along the directed sequence in the construction of $L_{P}$ it suffices to show that the bottom row is exact in the middle and that $\operatorname{im}\left(i^{\prime}\right) \subset \zeta\left(G^{\prime}\right)$, the center of $G^{\prime}$.

To see the latter, suppose $x, y, z \in G^{\prime}$ satisfy $x y=y x$ and $z^{n}=x$, for $n \in P^{\prime}$. Then $z^{n}=y z^{n} y^{-1}=\left(y z y^{-1}\right)^{n}$ and, hence, $z=y z y^{-1}$ because $n$th roots in $G^{\prime}$ are unique.

It remains to show that $\operatorname{ker}\left(v^{\prime}\right) \subset \operatorname{im}\left(i^{\prime}\right)$. Let $c: G^{\prime} \rightarrow S$ denote the cokernel of $i^{\prime}$. Note that $v^{\prime}$ factors uniquely through $S$. As $c \psi i=c i^{\prime} \varphi$ is trivial, $\psi c$ factors through $R$ via $\alpha$. Moreover, $\xi v=v^{\prime} \psi=\beta c \psi=\beta \alpha v$ and so $\xi=\beta \alpha$. We show that $\beta$ is an isomorphism.

Step 1: $P^{\prime}$-roots are unique in $S$. Let $\bar{x}, \bar{y} \in S$, and suppose $\bar{x}^{n}=\bar{y}^{n}$, for some $n \in P^{\prime}$. Then $x^{n} y^{-n}=i^{\prime}\left(a^{\prime}\right)$. Since $A$ is abelian, $A^{\prime}$ and $\operatorname{im}\left(i^{\prime}\right)$ are 
$P$-local. So pick $a \in \operatorname{im}\left(i^{\prime}\right)$ with $a^{n}=a^{\prime}$. Thus $x^{n}=a^{n} y^{n}=(a y)^{n}$ because $\operatorname{im}\left(i^{\prime}\right) \subset \zeta\left(G^{\prime}\right)$. As $P^{\prime}$-roots in $G^{\prime}$ are unique, $x=a y$ and so $\bar{x}=\bar{y}$ in $S$.

Step 2: $\alpha$ extends to an epimorphism $\gamma: \sqrt{ }_{P} R \rightarrow S$. The element $\alpha(v(g))$ has the unique $n$th roots $c\left(\psi(g)^{1 / n}\right)$. So $\gamma$ exists. It is onto because $G^{\prime}$ is generated by elements of the form $\psi(g)^{1 / n}$.

Step 3: $\beta$ is an isomorphism. We now have the commuting diagram of epimorphisms

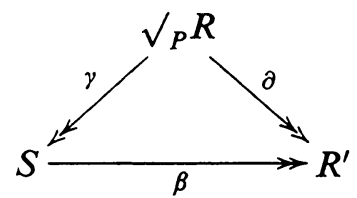

As $P^{\prime}$-roots in $S$ are unique, $\operatorname{ker}(\partial) \subset \operatorname{ker}(\gamma)$. By commutativity, $\operatorname{ker}(\gamma) \subset$ $\operatorname{ker}(\partial)$. The claim follows.

(vii) The restriction of $L_{P}$ to nilpotent groups is treated in $[19, \S 8]$. Thus the description of $\operatorname{ker}\left(\varepsilon_{P}\right)$ and $\operatorname{im}\left(\varepsilon_{P}\right)$ given in [11,13] applies.

We illustrate, by way of examples, how drastically different $P$-localization can act on an arbitrary group, as opposed to on a nilpotent group.

0.2 Example. (i) On the category of all groups $P$-localization is not an exact functor. Consider, for example, the symmetric group $S_{3}$ and the split exact sequence $C_{3} \mapsto S_{3} \rightarrow C_{2}$, where $C_{n}$ denotes the cyclic group of order $n$. Localizing away from the prime 2 yields $C_{3} \rightarrow 1 \rightarrow 1$, which is no longer exact. Still there is a description of the effect of $P$-localization on a semidirect product in terms of its effect on the factors; see [15].

(ii) There are groups, like the infinite alternating group, which become trivial if one inverts any given prime. To see this, recall that a $P$-local group is always $P^{\prime}$-torsion free. $A_{\infty}$ has torsion at every prime and has only the two trivial normal subgroups. Thus, if $P^{\prime} \neq \varnothing$, any homomorphism from $A_{\infty}$ to a $P$ local group must be trivial.

(iii) $P$-localizing a torsion free group can have a nontrivial kernel. A historic example is due to B. Neumann; see [2].

Despite these phenomena there is a nicely structured context within which $P$-localization of groups is well behaved; see $\S \S 2-7$.

\section{The $\mathscr{L}$-genus: Basic FACTS}

Let $\mathscr{C}$ be a category, and let $\left\{\left(L_{\mu}, \varepsilon_{\mu}\right)\right\}$ be a family of localizing functors on $\mathscr{C}$. Let $\left(S_{\mu}, D_{\mu}\right)$ be the orthogonal pair associated to $L_{\mu}$; i.e., $S_{\mu}$ is the class of morphisms $s \in \mathscr{C}$ for which $L_{\mu}(s)$ is an isomorphism, and $D_{\mu}$ consists of all objects $X$ in $\mathscr{C}$ for which $\varepsilon_{\mu}: X \rightarrow L_{\mu} X$ is an isomorphism; compare [1], see [9]. These pairs are partially ordered by

$$
\left(S_{\mu}, D_{\mu}\right) \geq\left(S_{\lambda}, D_{\lambda}\right) \text { if } D_{\mu} \supseteq D_{\lambda},
$$

in which case there is a natural transformation $L_{\mu} \rightarrow L_{\lambda}$ given by

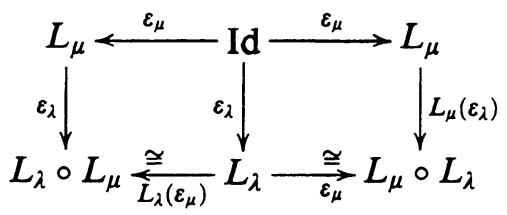


The arrow $\varepsilon_{\mu}$ at the bottom is an isomorphism because $D_{\lambda} \subset D_{\mu} . L_{\lambda}\left(\varepsilon_{\mu}\right)$ is seen to be an isomorphism by using universal properties of localization.

We write $\mathscr{L}$ for the family $\left\{\left(L_{\mu}, \varepsilon_{\mu}\right)\right\}$ together with the partial order described above. We regard $\mathscr{L}$ as a small category whenever this is convenient. Applying the functors in $\mathscr{L}$ to an object $X \in \mathscr{C}$ yields a diagram $\mathscr{L}(X)$ in $\mathscr{C}$, which is modeled on $\mathscr{L}$. We collect all diagrams in $\mathscr{C}$ which could conceivably arise in this way into a category denoted $\mathscr{L} \mathscr{C}$. An object $\underline{X}=\left\{u_{\lambda, \mu}: X_{\mu} \rightarrow X_{\lambda}\right\}$ of $\mathscr{L} \mathscr{C}$ is a diagram in $\mathscr{C}$ such that $X_{\mu} \in D_{\mu}$ and each arrow $u_{\lambda, \mu}$ in $\underline{X}$ is $L_{\lambda}$-localization; i.e., the diagram below commutes:

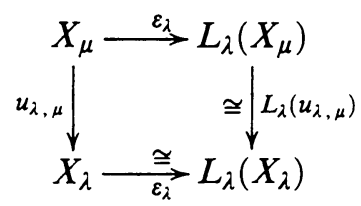

We refer to an object of $\mathscr{L} \mathscr{C}$ as an $\mathscr{L}$-diagram.

1.1 Definition. The genus of $X \in \mathscr{L} \mathscr{C}$ consists of all isomorphism classes of objects $X \in \mathscr{C}$ with $\mathscr{L}(X) \cong \underline{X}$. Objects $X$ and $Y$ of $\mathscr{C}$ belong to the same $\mathscr{L}$-genus if $\mathscr{L}(X) \cong \mathscr{L}(Y)$.

Next we establish a basic interaction between $\mathscr{L}$-genus and coproducts in $\mathscr{C}$. In special cases this interaction has already been observed by ad hoc methods and put to use; see, e.g., Bousfield [6].

1.2 Lemma. Suppose $\mathscr{C}$ is a category with finite coproducts. If $\mathscr{L}(X) \cong \mathscr{L}\left(X^{\prime}\right)$, then, for arbitrary $Y \in \mathscr{C}$,

$$
\mathscr{L}(X \sqcup Y) \cong \mathscr{L}\left(X^{\prime} \sqcup Y\right) \text {. }
$$

The proof of 1.2 makes use of the following elementary observation together with its Corollary 1.4 .

1.3 Observation. Let $L$ be a localizing functor on $\mathscr{E}$, and let $i: D \hookrightarrow \mathscr{C}$ be the inclusion of the full subcategory of $L$-local objects in $\mathscr{C}$. Suppose $F: \mathscr{J} \rightarrow D$ is a functor from a small category such that $\lim ^{\mathscr{E}}(i \circ F: \mathscr{J} \rightarrow \mathscr{C})$ exists as a colimit in $\mathscr{C}$. Then $\stackrel{\lim }{\longrightarrow} F$ exists as a colimit in $D$ and

$$
\stackrel{\lim }{\longrightarrow}^{D} F \cong L\left(\stackrel{\lim }{\longrightarrow}^{\mathscr{C}} i \circ F\right) \text {. }
$$

1.4 Corollary. As in 1.3 let $L: \mathscr{C} \rightarrow D$ be a localizing functor, and let $G: \mathscr{J} \rightarrow$ $\mathscr{C}$ be a functor. If $\mathscr{C}$ is cocomplete, so is $D$ and

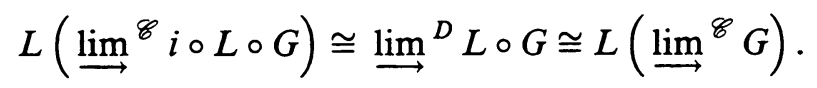

Proof. The left-hand side isomorphism is 1.3. The right-hand side isomorphism holds because $L$ commutes with colimits ( $L$ is left adjoint to the inclusion $D \hookrightarrow \mathscr{C}$ ).

Proof of 1.2. Corollary 1.4 yields for $L=L_{\mu}$ :

$$
L(X \sqcup Y) \cong L(L(X) \sqcup L(Y)) \cong L\left(L\left(X^{\prime}\right) \sqcup L(Y)\right) \cong L\left(X^{\prime} \sqcup Y\right) .
$$

To see that these isomorphisms of objects in $\mathscr{C}$ form an isomorphism of diagrams

$$
\mathscr{L}(X \sqcup Y) \cong \mathscr{L}\left(X^{\prime} \sqcup Y\right)
$$


in $\mathscr{L} \mathscr{C}$, consider the arrows modeled on $\mu \geq \lambda$. By hypothesis we have a commuting diagram:

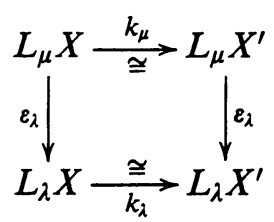

Commutativity of the corresponding diagram between $\mathscr{L}(X \cup Y)$ and $\mathscr{L}\left(X^{\prime} \cup Y\right)$ can be read off from the diagram below whose individual rectangles are known to commute:

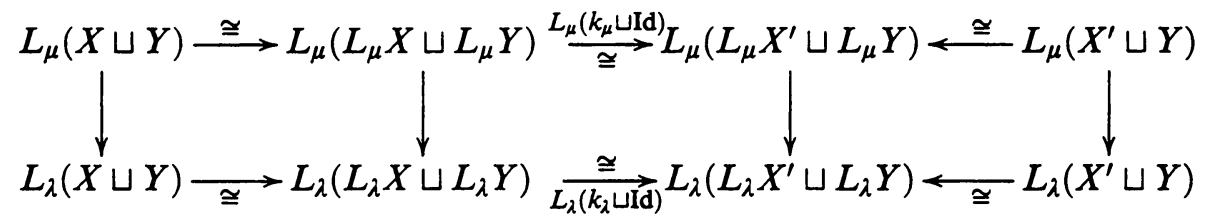

1.5 Corollary. If $\mathscr{C}$ has finite coproducts, then there is defined "addition" of $\mathscr{L}$. genera

$$
[X]_{\mathscr{L}}+[Y]_{\mathscr{L}}:=\left[X \sqcup Y_{\mathscr{L}} .\right.
$$

1.6 Definition. Suppose $\mathscr{C}$ has a 0 -object. An element $X \in \mathscr{C}$ is $\mathscr{L}$-trivial if $L_{\mu} X=0$ for all $\mu$; i.e. if $\mathscr{L}(X)$ is a 0 -object in $\mathscr{L} \mathscr{C}$.

1.7 Remark. Suppose $\mathscr{C}$ is a category with finite coproducts. Then isomorphism classes of $\mathscr{C}$ form an abelian "semigroup" $\mathscr{E}$, and $\mathscr{L}$-genera in $\mathscr{C}$ form a quotient $\mathscr{L}_{U}$ of $\mathscr{C}_{U}$. If $\mathscr{C}$ has a 0 -object, $\mathscr{C}_{U}$ is an "abelian monoid" and the kernel of the "monoid" epimorphism $\mathscr{E}_{U} \rightarrow \mathscr{L}_{L}$ consists of all $\mathscr{L}$-trivial objects.

Now suppose that $\mathscr{E}$ has inverse limits. By $\mathscr{C}(A, B)$ we denote the set of morphisms from $A$ to $B$ in the category $\mathscr{C}$.

1.8 Proposition. The functor $\mathscr{L}: \mathscr{C} \rightarrow \mathscr{L} \mathscr{C}$ is left adjoint to the functor $\underline{\mathrm{lim}}$ : $\mathscr{L} \mathscr{C} \rightarrow \mathscr{C}$.

Proof. We need to show that, for any object $A$ of $\mathscr{C}$ and any $\mathscr{L}$-diagram $\underline{X}$ in $\mathscr{L} \mathscr{C}$, there is a natural bijection

$$
\gamma: \mathscr{C}(A, \underline{\lim } \underline{X}) \rightarrow \mathscr{L} \mathscr{C}(\mathscr{L}(A), \underline{X}) .
$$

The universal property of $P$-localization determines, for every $f: A \rightarrow \underline{\lim } \underline{X}$, a unique commutative diagram:

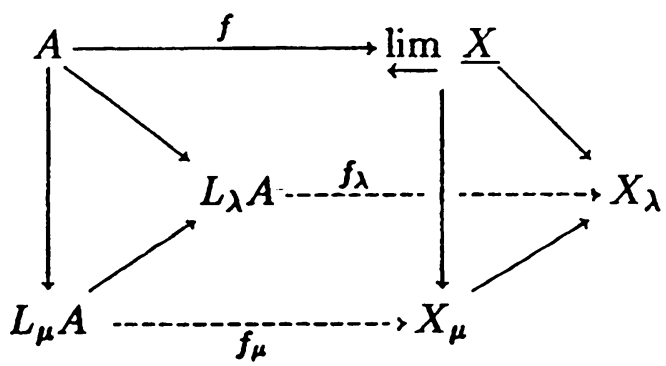


Thus $\gamma(f)$ is defined. The inverse of $\gamma$ is given by $\underline{\text { lim }}$ applied to a morphism $\mathscr{L}(A) \rightarrow \underline{X}$. The required naturality properties of $\gamma$ follow from the universal property of localization and $\underline{\mathrm{lim}}$.

1.9 Remark. If $\mathscr{C}$ has only weak inverse limits for the diagrams modeled on $\mathscr{L}$ (which is likely to happen in the homotopy category of CW-spaces), then $\gamma$ is still defined as above. It will still be onto, but will, in general, fail to be $1-1$.

Here are some example of familiar concepts considered from the unifying point of view of $\mathscr{L}$-genus, respectively $\mathscr{L}$-completion, using a suitable system of localizing functors $\mathscr{L}$.

1.10 Example. Let $\mathscr{G}$ be the category of groups and, for $c \geq 0$, let $\mathscr{N}_{c}$ be the category of groups which are nilpotent of order $\leq c$. Killing commutators of order $>c$ in a group gives an idempotent functor

$$
N_{c}: \mathscr{G} \rightarrow \mathscr{N}_{c}
$$

which is left adjoint to the inclusion $\mathscr{N}_{c} \rightarrow \mathscr{G}$. So $N_{c}$ is a localizing functor. The functors $N_{c}$ form a tower

$$
\nu=\cdots \rightarrow N_{c+1} \rightarrow N_{c} \rightarrow \cdots \rightarrow N_{0} .
$$

The $\nu$-completion of a group $G$ is known as the nilpotent completion of $G$.

1.11 Example. Let $C W_{*}$ be the homotopy category of based connected CWcomplexes and let $C W_{*}^{[k]}$ be the full subcategory of $(k+1)$-anticonnected spaces $\left(\pi_{n} X=0\right.$, if $\left.n \geq k+1\right)$. Killing homotopy groups in dimensions $\geq(k+1)$ in a space gives an idempotent functor

$$
P o_{k}: C W_{*} \rightarrow C W_{*}^{[k]}
$$

which is left adjoint to the inclusion $C W_{*}^{[k]} \rightarrow C W_{*}$. So $P o_{k}$ is a localizing functor. The functors $P_{k}$ form a tower

$$
\mathrm{Po}=\cdots \rightarrow P o_{k+1} \rightarrow P o_{k} \rightarrow \cdots \rightarrow P o_{0} .
$$

The $P o$-diagram of a space $X$ is known as the Postnikov tower of $X$. The natural map $X \rightarrow$ ho invlim $P o(X)$ is a homotopy equivalence. In particular, the $P o$-genus of a space is a single homotopy type. This example has been generalized in recent work by Bousfield [6].

1.12 Example. Let $P o_{k}$ be as above and form the "discrete" system of localizing functors

$$
P o_{\text {disc }}=\cdots P o_{k+1} P o_{k} \cdots P o_{0} .
$$

The $P o_{\text {disc }}$-genus of a space $X$ is known as the set of "spaces of the same $n$-type" as $X$, for all $n$, denoted $\operatorname{SNT}(X)$; see Wilkerson [21].

1.13 Example. For a prime $p$, let $\mathscr{G}_{p}$ be the category of groups which are uniquely radicable at all primes $q \neq p$. Let

$$
L_{p}: \mathscr{G} \rightarrow \mathscr{G}_{p}
$$


be the localizing functor described in $\S 0$. The functors $L_{p}$ form the discrete system

$$
\mathscr{L}=L_{2} L_{3} \cdots L_{p} \cdots .
$$

When restricted to nilpotent groups, the $\mathscr{L}$-genus is known as the extended genus. When restricted to finitely generated nilpotent groups, the $\mathscr{L}$-genus of such a group $G$ is known as "the" genus of $G$; see [13]. An arbitrary nilpotent group $H$ is of the same Mislin genus as the finitely generated group $G$ if $\mathscr{L}(G) \cong \mathscr{L}(H)$.

1.14 Example. For a prime $p$, let $\lambda_{p}$ be a $p$-localizing functor on the category of (based) connected CW-complexes which extends $p$-localization of nilpotent spaces; e.g., Bousfield's $H_{*}\left(\bullet ; \mathbb{Z}_{(p)}\right)$-localization [5] or its maximally twistsensitive parent, described in [8], are good choices. The functors $\lambda_{p}$ form a system

$$
\Lambda=\lambda_{2} \lambda_{3} \cdots \lambda_{p} \cdots .
$$

When restricted to nilpotent spaces of finite type (each homotopy group is finitely generated), the $\Lambda$-genus of such a space $X$ is known as "the" genus of $X$; see [13]. An arbitrary space $Y$ is of the same Mislin genus as the nilpotent space $X$ of finite type if $\Lambda(X) \cong \Lambda(Y)$.

1.15 Example. Let $C l:=P o_{\text {disc }} \cup \Lambda$. Each member in the $C l$-genus of a nilpotent space $X$ is referred to as a clone of $X$; see [14].

\section{2. $\mathscr{L}$-DIAGRAMS OF GROUPS CORRESPONDING TO A PARTITION OF PRIMES}

Let $Q$ be a set of primes. A partition $\mathscr{Q}=\left\{Q ; P_{1}, P_{2}, \ldots\right\}$ of all primes relative to $Q$ consists of sets of primes $P_{\mu}$ containing $Q$ such that each prime not in $Q$ belongs to precisely one of the sets $P_{\mu}$. Such a partition determines the diagram of natural transformations between localizing functors displayed below.

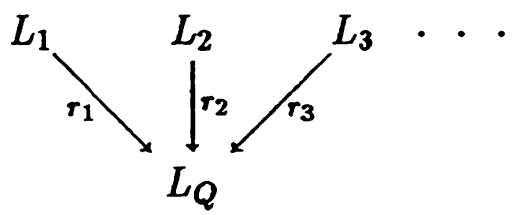

Here $L_{\mu}:=L_{P_{\mu}}$ is the $P_{\mu}$-localizing functor on the category of groups $\mathscr{G}$. To avoid spurious considerations, we assume throughout that $P_{1}$ does not contain all primes.

According to $\S 1$ we form the category $\mathscr{L} \mathscr{G}$ of $\mathscr{L}$-diagrams in $\mathscr{G}$ and proceed to describe the relationship between the categories $\mathscr{G}$ and $\mathscr{L} \mathscr{G}$ given by the pair of adjoint functors $\mathscr{L}: \mathscr{G} \rightarrow \mathscr{L} \mathscr{G}$ and $\underline{\lim }: \mathscr{L} \mathscr{G} \rightarrow \mathscr{G}, \underline{G} \mapsto \underline{\lim } \underline{G}$. We often write $\underline{G}^{\sim}$ for $\lim ^{G}$. The main result of this section is

2.1 Theorem. Let $\mathscr{L}$ be the family of localizing functors on the category of groups corresponding to a partition $\mathscr{Q}=\left\{Q ; P_{1}, \ldots\right\}$ of the set of all primes. Let $G$ be any group such that

(i) $\operatorname{im}\left(\varepsilon_{Q}: G \rightarrow L_{Q} G\right)=\bigcap \operatorname{im}\left(r_{\mu}: L_{\mu} G \rightarrow L_{Q} G\right)$; 
(ii) $\operatorname{ker}\left(\varepsilon_{Q}\right)$ is the restricted product $\Pi^{w} K_{\mu}$, where $K_{\mu}$ is $P_{\mu}$-local and $L_{\nu} K_{\mu}=1$ if $\mu \neq \nu$.

Then the natural homomorphism $c: G \rightarrow \lim \mathscr{L}(G)$ is a monomorphism. Moreover, $c$ is an isomorphism if and only if all but at most finitely many of the groups $K_{\mu}$ are trivial.

Classes of groups satisfying the hypotheses of Theorem 2.1 are described in $\S 3$. Auxiliary results leading to a proof of 2.1 follow.

2.2 Lemma. Let $G$ be a group and let $R:=\operatorname{im}\left(\varepsilon_{Q}: G \rightarrow L_{Q} G\right)$. Then the inclusion of $R$ in $L_{Q} G$ induces an isomorphism $L_{Q} R \rightarrow L_{Q} G$.

Proof. Consider the commutative diagram below, where $L_{Q^{\alpha}}$ is an epimorphism, using 0.1(iii):

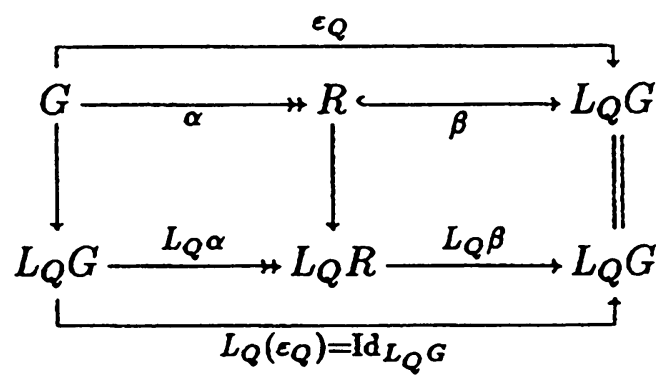

Further, $L_{Q} \alpha$ is a monomorphism because $\left(L_{Q} \beta\right)\left(L_{Q} \alpha\right)=\operatorname{Id}_{L_{Q}} G$. So $L_{Q} \beta=\left(L_{Q^{\alpha}}\right)^{-1}$ is an isomorphism.

2.3 Lemma. Let $Q \subset P$ be sets of primes and suppose $G$ is a P-local group. Then the kernel and the image of the $Q$-localizing homomorphism $G \rightarrow L_{Q} G$ are P-local groups.

Proof. Let $n \in P^{\prime}$ and $x \in \operatorname{ker}\left(G \rightarrow L_{Q} G\right)$. Then there is a unique element $y \in G$ with $x=y^{n}$. But then

$$
1=\varepsilon_{Q}(x)=\varepsilon_{Q}\left(y^{n}\right)=\left(\varepsilon_{Q}(y)\right)^{n} .
$$

Hence $\varepsilon_{Q}(y)=1$ because $P^{\prime}$-roots in $L_{Q} G$ are unique.

$R:=\operatorname{im}\left(G \rightarrow L_{Q} G\right)$ is $P$-local because (1) the $n$th power operation, $n \in$ $P^{\prime}$, on $R$ is onto (it is onto in $G$ ), and (2) it is $1-1$ as $R$ is contained in the $P$-local group $L_{Q} G$.

2.4 Proposition. Let $\underline{G}=\left\{r_{\mu}: G_{\mu} \rightarrow G_{Q}\right\}$ be an $\mathscr{L}$-diagram in $\mathscr{G}$. For each index $\mu$, let $K_{\mu}:=\operatorname{ker}\left(r_{\mu}\right), I_{\mu}:=\operatorname{im}\left(r_{\mu}\right)$, and put $I:=\bigcap I_{\mu}$. If the inclusion of $I$ in $G_{Q}$ induces an isomorphism $L_{Q} I \rightarrow G_{Q}$ and if $L_{Q} K_{\mu}=1$, for all $\mu$, then the following hold.

(i) The sequence of $\mathscr{L}$-diagrams $\underline{K} \mapsto \underline{G} \rightarrow \underline{I}$ is exact at every $\mu$; here $\underline{K}=\left\{K_{\mu} \rightarrow 1\right\}$ and $\underline{I}=\left\{I_{\mu} \hookrightarrow G_{Q}\right\}$.

(ii) The corresponding sequence of $\mathscr{L}$-completions $\underline{K}^{\sim} \hookrightarrow \underline{G}^{\sim} \rightarrow \underline{I}^{\sim}$ is exact. 
Proof. From the hypotheses we have the commutative diagram below.

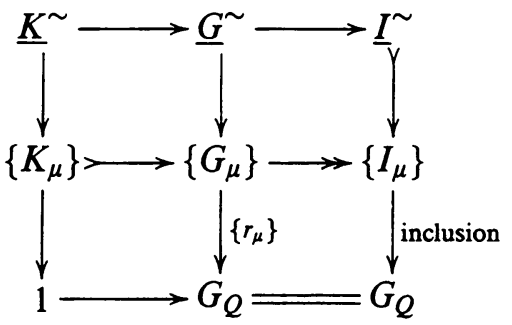

The groups $K_{\mu}$ are $P_{\mu}$-local by 2.3. So maps $\left\{K_{\mu} \rightarrow 1\right\}$ form an $\mathscr{L}$-diagram because $L_{Q} K_{\mu}=1$. The inclusions $\left\{I_{\mu} \hookrightarrow L_{Q} I_{\mu}=G_{Q}\right\}$ form an $\mathscr{L}$-diagram by 2.2. Thus the top row is a sequence of $\mathscr{L}$-completions. It is exact in the left-hand and center positions using general results about inverse limits of sets; see $[4$, pp. $194 \mathrm{ff}]$.

To see that the map $\underline{G}^{\sim} \rightarrow \underline{I}^{\sim}$ is onto, note first that we may choose $\underline{I}^{\sim}=I$ and $\underline{I}^{\sim} \rightarrow I_{\mu}$ as the inclusion $I \rightarrow I_{\mu}$. Further, the image of any of the composites $\underline{G}^{\sim} \rightarrow G_{\mu} \rightarrow G_{Q}$ is equal to $I$. The claim follows from the commutativity of the diagram.

2.5 Lemma. Let $\underline{G}=\left\{r_{\mu}: G_{\mu} \rightarrow G_{Q}\right\}$ be an $\mathscr{L}$-diagram and define $I, I_{\mu}, K_{\mu}$ as in 2.4. If, for each $\mu, I \hookrightarrow I_{\mu}$ induces an isomorphism $L_{\mu} I \rightarrow I_{\mu}$ and if $L_{Q}\left(K_{\mu}\right)=1$, then

(i) $\operatorname{im}\left(L_{\mu}\left(\underline{G}^{\sim}\right) \rightarrow G_{\mu}\right)$ contains $K_{\mu}$;

(ii) $u: L_{\mu}\left(\underline{G}^{\sim}\right) \rightarrow G_{\mu}$ is onto.

If, in addition, $K_{\mu} \neq 1$ for at most finitely many $\mu$, then

(iii) $L_{\mu}\left(\Pi K_{\mu}\right) \cong K_{\mu}$ and $K_{\mu} \rightarrow L_{\mu}\left(\underline{G}^{\sim}\right)$ is a monomorphism;

(iv) $L_{\mu}\left(\underline{G}^{\sim}\right) \rightarrow G_{\mu}$ is an isomorphism if and only if the sequence below is exact:

$$
1 \rightarrow L_{\mu}\left(\prod K_{\mu}\right) \longrightarrow L_{\mu}\left(\underline{G}^{\sim}\right) \rightarrow L_{\mu} I \rightarrow 1 .
$$

Proof. All of the relevant information is assembled in the commutative diagram below in which the front rows are exact, using 2.4:

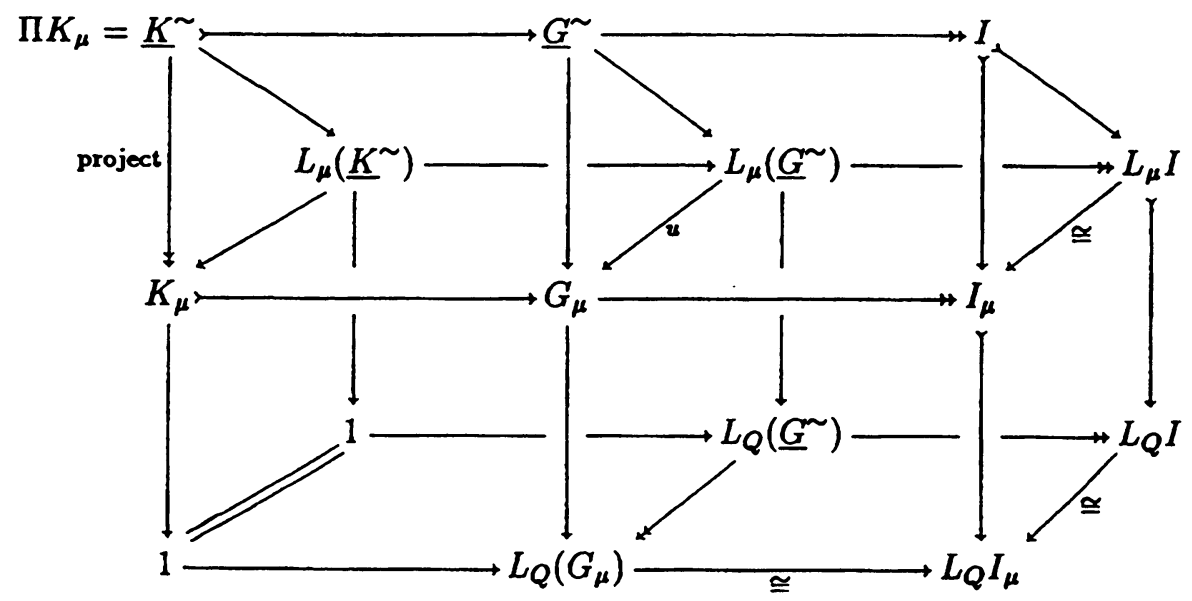

Now (i) follows because $\underline{K}^{\sim} \rightarrow K_{\mu}$ is onto. (ii) is a consequence, using exactness of $K_{\mu} \longmapsto G_{\mu} \rightarrow I_{\mu}$. To see (iii), recall that localization commutes 
with finite products; see $0.1(\mathrm{v})$. Hence $L_{\mu}\left(\underline{K}^{\sim}\right) \rightarrow K_{\mu}$ is an isomorphism. To see (iv), read off from commutativity

$$
u^{-1}\left(K_{\mu}\right)=\operatorname{ker}\left(L_{\mu}\left(\underline{G}^{\sim}\right) \stackrel{u}{\rightarrow} G_{\mu} \rightarrow I_{\mu}\right)=\operatorname{ker}\left(L_{\mu}\left(\underline{G}^{\sim}\right) \rightarrow L_{\mu} I\right)
$$

and use the 5-lemma.

2.6 Lemma. If $\varepsilon_{Q}: G \rightarrow L_{Q} G$ is a monomorphism and if

$$
\operatorname{im}\left(\varepsilon_{Q}: G \mapsto L_{Q} G\right)=\bigcap \operatorname{im}\left(r_{\mu}: L_{\mu} G \rightarrow L_{Q} G\right)=I,
$$

then $c: G \rightarrow \mathscr{L}(G)^{\sim}$ is an isomorphism.

Proof. $\mathscr{L}(G)^{\sim}=I$ because each $r_{\mu}$ is a monomorphism. The claim follows.

Proof of 2.1. We have the commutative diagram:

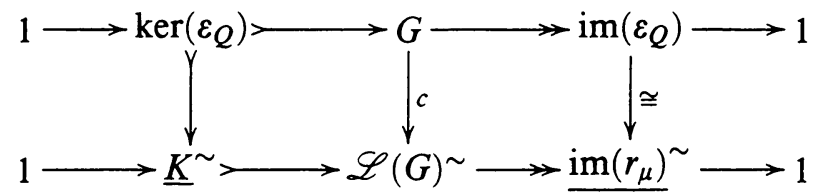

The bottom row is exact. To see this, note that

$$
L_{Q}\left(\bigcap \operatorname{im}\left(r_{\mu}: L_{\mu} G \rightarrow L_{Q} G\right)\right)=L_{Q}\left(\operatorname{im}\left(\varepsilon_{Q}: G \rightarrow L_{Q} G\right)\right)=L_{Q} G,
$$

by 2.2. So the bottom row is exact by 2.4. The map $\operatorname{ker}\left(\varepsilon_{Q}\right) \rightarrow \underline{K}^{\sim}$ is the inclusion of the weak product $\Pi^{w} K_{\mu}$ into the cartesian product $\Pi K_{\mu}$, because localization commutes with restricted products. The vertical arrow on the right is an isomorphism by 2.6. Thus $G \rightarrow \mathscr{L}(G)^{\sim}$ is a monomorphism using commutativity of the diagram. Finally, if all but finitely many of the groups $K_{\mu}$ are trivial then the vertical arrow on the left is an isomorphism. So $c$ is an isomorphism by the 5-lemma.

\section{The CATEgory $\mathscr{G}_{\mathscr{Q}}^{1}$}

Given a relative partition of primes $\mathscr{Q}=\left\{Q ; P_{1}, P_{2}, \ldots\right\}$, let $\mathscr{G}_{\mathscr{Q}}^{1}$ denote the category of all groups $G$ satisfying

(i) $K_{\mu}:=\operatorname{ker}\left(L_{P_{\mu}} G \rightarrow L_{Q} G\right)$ consists of $\left(P_{\mu}-Q\right)$-torsion elements;

(ii) $\operatorname{ker}\left(G \rightarrow L_{Q} G\right)$ consists of $Q^{\prime}$-torsion elements;

(iii) if $P \in \mathscr{Q}$ and $x \in L_{P} G$, there exists $n \in P^{\prime}$ such that $x^{n}$ is contained in the image of the localizing map $G \rightarrow L_{P} G$.

Groups in $\mathscr{G}_{\mathscr{Q}}^{1}$ behave nicely with respect to $P$-localization. In particular, Theorem 2.1 has a stronger reformulation for such groups; see 4.1. An outline of their key properties follows.

3.1 Theorem. If $P \in \mathscr{Q}$, then $L_{P}$ is an exact functor on $\mathscr{G}_{\mathscr{Q}}^{1}$.

3.2 Theorem. $\mathscr{G}_{\mathscr{Q}}^{1}$ is closed under directed colimits.

3.3 Theorem. Let $N \stackrel{\alpha}{\rightarrow} G \stackrel{\beta}{\rightarrow} H$ be a central extension.

(i) If $N, H$ are in $\mathscr{G}_{\mathbb{Q}}^{1}$ and if, for $P \in \mathscr{Q}, L_{P} \alpha$ is a monomorphism, then $G$ is in $\mathscr{E}_{\mathscr{Q}}$.

(ii) If $N, G$ are in $\mathscr{G}_{\mathbb{Q}}^{1}$, so is $H$. 
3.4 Remark. Basic properties of localization (see $\S 0$ ) imply the following statements.

(i) If $G$ is a finite group and if $Q$ contains all primes dividing the order of $G$, then $G$ belongs to $\mathscr{G}_{\mathscr{Q}}^{1}$, for every partition of the set of primes relative to $Q$.

(ii) If $G$ is a torsion group which, modulo its Fitting subgroup, is $Q$ torsion, then $G$ belongs to every one of the categories $\mathscr{G}_{\mathscr{Q}}^{1}$ with $\mathscr{Q}=$ $\left\{Q ; P_{1}, P_{2}, \ldots\right\}$.

(iii) $Q$-local groups belong to $\mathscr{G}_{\mathscr{Q}}^{1}$, if $\mathscr{Q}=\left\{Q ; P_{1}, P_{2}, \ldots\right\}$.

(iv) Nilpotent groups and directed colimits of nilpotent groups belong to every one of the categories $\mathscr{G}_{\mathscr{Q}}^{1}$. This follows from 3.2 (directed colimits of nilpotent groups are usually called "locally nilpotent groups"; compare [12]).

(v) There are groups, like the infinite alternating group, which do not belong to any of the categories $\mathscr{G}_{\mathscr{Q}}^{1}$; compare 0.2 (ii).

3.5 Remark. If $G$ belongs to $\mathscr{G}_{\mathscr{Q}}^{1}$ and $P \in \mathscr{Q}$, then the epimorphism $\sqrt{ }_{P} G \rightarrow$ $L_{P} G$ induces an isomorphism $U_{P} \sqrt{ }_{P} G \rightarrow L_{P} G$. This follows by feeding axiom (iii) on $\mathscr{G}_{\mathscr{Q}}^{1}$ into the construction of $P$-localization described in $\S 0$.

3.6 Remark. Let $G$ be a group such that, for each $\mu,\left(P_{\mu}-Q\right)$-torsion elements form a normal subgroup $T_{\mu}[G]$ of $G$. Let $T_{Q^{\prime}}[G]$ denote the subgroup of $G$ generated by $Q^{\prime}$-torsion elements. Then

(i) $T_{Q^{\prime}}[G] \triangleleft G$;

(ii) $T_{Q^{\prime}}[G] \cong \prod_{\mu}^{w} T_{\mu}[G]$ is a restricted product.

Proof. Part (i) is elementary. As for part (ii), a finite collection $T_{\mu_{1}}[G], \ldots$, $T_{\mu_{k}}[G]$ generates a product $T_{\mu_{1}}[G] \times \cdots \times T_{\mu_{k}}[G] . T_{Q^{\prime}}[G]$ is the directed colimit of such products. This implies the claim.

Proof of Theorem 3.1. An exact sequence $N \mapsto G \rightarrow R$ gives rise to the following commuting diagram:

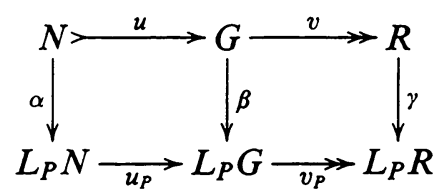

where $v_{P}$ is onto, using 0.1 (ii). Now suppose that the groups in the top row are in $\mathscr{G}_{\mathscr{Q}}$ and choose $P \in \mathscr{Q}$. Functorial properties of $L_{P}$ imply that $v_{P} \circ u_{P}$ is the trivial homomorphism. Thus we need to show that $\operatorname{ker}\left(v_{P}\right) \subset \operatorname{im}\left(u_{P}\right)$.

If $v_{P}(x)=1$, choose $n \in P^{\prime}$ such that $x^{n}=\beta(g)$, for some $g \in G$. It follows that $v(g) \in \operatorname{ker}(\gamma)$ and so, $v(g)^{m}=1$, for some $m \in P^{\prime}$. But then $g^{m} \in \operatorname{ker}(v)$. So there is $y \in N$ with $u(y)=g^{m}$. Therefore, $x=$ $u_{P}\left(\alpha(y)^{1 / m n}\right) \in \operatorname{im}\left(u_{P}\right)$. So the sequence is exact in the middle.

It remains to show that $u_{P}$ is a monomorphism. If $u_{P}(x)=1$, pick $n \in P^{\prime}$ such that $x^{n}=\alpha(y)$. Then

$$
\beta \circ u(y)=u_{P} \circ \alpha(y)=u_{P}\left(x^{n}\right)=1 .
$$


Consequently $u(y)$ and hence $y$ are $P^{\prime}$-torsion. So $\alpha(y)=1=x^{n}$. So $x=1$ because $n$th roots are unique in $L_{P} N$.

Proof of 3.2. Let $\bar{G}$ be a directed diagram in $\mathscr{G}_{\mathscr{Q}}$. Given a set of primes $P$ containing $Q$, fit each group $G_{\lambda}$ in $\bar{G}$ into an exact sequence

$$
1 \rightarrow N_{\lambda} \nrightarrow G_{\lambda} \rightarrow R_{\lambda} \rightarrow 1,
$$

where $N_{\lambda}$ is the kernel of the $P$-localizing map $G_{\lambda} \rightarrow L_{P} G_{\lambda}$. The directed diagram $\bar{G}$ yields a directed diagram of such short exact sequences, and the resulting sequence of colimits is exact (see [10]):

$$
1 \rightarrow \underline{\lim _{\longrightarrow}} \bar{N} \hookrightarrow \lim _{\longrightarrow} \bar{G} \rightarrow \lim _{\longrightarrow} \bar{R} \rightarrow 1 .
$$

We obtain the commuting diagram below:

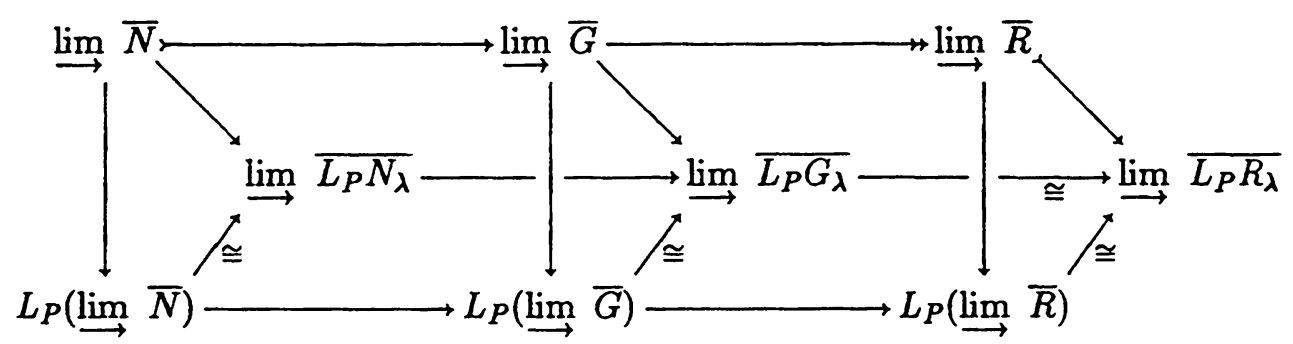

Each group $N_{\lambda}$ consists of $P^{\prime}$-torsion elements, and so $L_{P} N_{\lambda}$ is trivial. Further, $G_{\lambda} \rightarrow R_{\lambda}$ induces an isomorphism $L_{P} G_{\lambda} \rightarrow L_{P} R_{\lambda}$, by 2.2 . To explain the other indicated isomorphisms, recall that localization commutes with directed colimits; see 0.1 (iv). Each map $R_{\lambda} \rightarrow L_{P} R_{\lambda}$ is a monomorphism. Hence $\stackrel{\lim }{\longrightarrow} \rightarrow \underline{\lim } \underline{L_{P} R_{\lambda}}$ is a monomorphism. Therefore

$$
\stackrel{\lim }{\longrightarrow} \bar{N}=\operatorname{ker}\left(\lim _{\longrightarrow} \bar{G} \rightarrow L_{P}\left(\lim _{\longrightarrow} \bar{G}\right)\right)
$$

Moreover, $\lim \bar{N}$ consists of $P^{\prime}$-torsion elements because it is a union of such. Next assume $x=\left[x_{\lambda}\right]_{P} \in L_{P}(\underset{\lim }{\vec{G}})$. Via the indicated isomorphism $x$ corresponds to $y=\left[y_{\lambda P}\right] \in \underset{\lim }{\longrightarrow} \overrightarrow{L_{P} G_{\lambda}}$. As $G_{\lambda} \in \mathscr{G}_{\mathbb{Q}}^{1}$, there exist $n \in P^{\prime}$ with $\left(y_{\lambda P}\right)^{n} \in \operatorname{im}\left(G_{\lambda} \rightarrow L_{P} G_{\lambda}\right)$. It follows that $x^{n} \in \operatorname{im}\left(\lim _{\longrightarrow} \bar{G} \rightarrow L_{P} \stackrel{\lim }{\longrightarrow}\right)$. Thus the colimit belongs to $\mathscr{G}_{\mathscr{Q}}$.

Proof of 3.3. Given $P \in \mathscr{Q}, P$-localization yields the commutative diagram below:

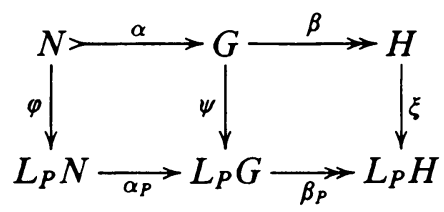

From 0.1 (vi) and its proof we know that the bottom row is exact in the middle and that $\operatorname{im}\left(\alpha_{P}\right) \subset \zeta\left(L_{P} G\right)$. Suppose $N, H \in \mathscr{G}_{\mathcal{E}}^{1}$. To see that $\operatorname{ker}(\psi)$ consists of $P^{\prime}$-torsion elements, suppose $\psi(x)=1$. Then $\beta(x) \in \operatorname{ker}(\xi)$ and, therefore, $\beta(x)^{n}=1$ for some $n \in P^{\prime}$. Consequently, $x^{n}=\alpha(y)$, for some $y \in N$. As

$$
\alpha_{P} \varphi(y)=\psi \alpha(y)=\psi\left(x^{n}\right)=1,
$$


we see that $y \in \operatorname{ker}(\varphi)$. So $y^{m}=1$ for some $m \in P^{\prime}$. So $x^{n m}=1$, and we see that $x$ is $P^{\prime}$-torsion.

Now let $x \in L_{P} G$. We are looking for $n \in P^{\prime}$ with $x^{n} \in \operatorname{im}(\psi)$. Pick $r \in P^{\prime}$ with $\beta_{P}(x)^{r}=\xi(h)=\xi \beta(y)$, for suitable choices of $h \in H$ and $y \in G$. So we have $\beta_{P}\left(x^{r}\right)=\beta_{P} \psi(y)$ or $\beta_{P}\left(x^{r} \psi(y)^{-1}\right)=1$. Therefore $x^{r} \psi(y)^{-1}=\alpha_{P}(a)$, which yields $x^{r}=\alpha_{P}(a) \psi(y)$. Pick $s \in P^{\prime}$ with $a^{s}=\varphi(b) \in \operatorname{im}(\varphi)$. Then

$$
x^{r s}=\alpha_{P}(a)^{s} \psi(y)^{s}=\psi(\varphi(b)) \psi\left(g^{s}\right)=\psi\left(\varphi(b) y^{s}\right)
$$

belongs to $\operatorname{im}(\psi)$. We have shown that $G \in \mathscr{G}_{\mathscr{Q}}^{1}$.

Suppose now that $N, G \in \mathscr{G}_{\mathscr{Q}}^{1}$. If $\xi(x)=1$, pick $g \in G$ with $\beta(g)=x$. We have $\psi(g)=\alpha_{P}(a)$ by exactness. Pick $n \in P^{\prime}$ with $a^{n}=\varphi\left(a_{1}\right)$ and observe $\psi(g)^{n}=\alpha_{P} \varphi\left(a_{1}\right)=\psi \alpha\left(a_{1}\right)$ and so $g^{n} \alpha\left(a_{1}\right)^{-1} \in \operatorname{ker}(\psi)=T_{P^{\prime}}[G]$. Pick $m \in P^{\prime}$ with $1=\left(g^{n} \alpha\left(a_{1}\right)^{-1}\right)^{m}=g^{n} \alpha\left(a_{1}\right)^{-m}$, i.e. $g^{n}=\alpha\left(a_{1}\right)^{m}$. So, we see $1=\beta\left(g^{n}\right)=x^{n}$. Hence $x \in T_{P^{\prime}}[H]$.

Now let $x=\beta_{P}(y) \in L_{P} H$. Pick $n \in P^{\prime}$ with $y^{n}=\psi(g)$. Then

$$
x^{n}=\beta_{P} \psi(g)=\xi(\beta(g)) \in \operatorname{im}(\xi) .
$$

\section{4. $\mathscr{L}$-DIAGRAMS IN $\mathscr{G}_{\mathscr{Q}}^{1}$}

For groups in $\mathscr{G}_{\mathscr{Q}}^{1}$ (see the previous section) our main local global result, Theorem 2.1, takes the following simple form.

4.1 Theorem. Given a partition $\mathscr{Q}=\left\{Q ; P_{1}, P_{2}, \ldots\right\}$ of the set of all primes relative to $Q$, let $\mathscr{L}=\left\{r_{\mu}: L_{\mu} \rightarrow L_{Q}\right\}$ be the corresponding diagram of localizing functors. If $G \in \mathscr{G}_{\mathscr{Q}}^{1}$, then the natural map $c: G \rightarrow \mathscr{L}(G)^{\sim}$ is a monomorphism. Moreover, $c$ is an isomorphism if and only if $G$ has $Q^{\prime}$-torsion at only finitely many of the sets $P_{\mu}$.

Proof. Feed 3.6 and 4.2 below into 2.1 .

4.2. Lemma. For a partition $\mathscr{Q}=\left\{Q ; P_{0}, P_{1}, \ldots\right\}$ of the set of primes and $a$ group $G \in \mathscr{G}_{\mathbb{Q}}^{1}$, we have

$$
\operatorname{im}\left(\varepsilon_{Q}: G \rightarrow L_{Q} G\right)=\bigcap \operatorname{im}\left(r_{\mu}: L_{\mu} G \rightarrow L_{Q} G\right) .
$$

Proof. $\operatorname{im}\left(\varepsilon_{Q}\right) \subset \bigcap_{\mu} \operatorname{im}\left(r_{\mu}\right)$, because $\varepsilon_{Q}=r_{\mu} \circ \varepsilon_{\mu}$, for all $\mu$. To see the converse inclusion, suppose $x \in \bigcap_{\mu} \operatorname{im}\left(r_{\mu}\right)$. Let $n \geq 1$ be the least integer such that $x^{n}$ belongs to the image of $\varepsilon_{Q}$. If $n=1$, the claim is proven. Otherwise $n=n_{1} \cdots n_{k}$ with integers $n_{i}>1$ belonging to the multiplicative closure of $\left(P_{\mu_{i}}-Q\right)$. Pick $g \in G$ with $\varepsilon_{Q}(g)=x^{n}$. Let $y \in L_{\mu_{1}} G$ be such that $x=r_{\mu_{1}}(y)$ and such that there exists $m \in\left(P_{\mu_{1}}\right)^{\prime}$ with $y^{m}=\varepsilon_{\mu_{1}}(h)$, for some $h \in G$. Then $c:=\operatorname{gcd}(m, n) \leq n / n_{1}<n$. Let $a, b \in \mathbb{Z}$ with $a m+b n=c$. Then $\varepsilon_{Q}\left(h^{a} g^{b}\right)=x^{c}$, which contradicts the minimality of $n$.

The following examples may help to illustrate the content of Theorem 4.1. Less trivial applications are contained in $\S \S 5,6$, and 7.

4.3 Example. Let $G:=\bigoplus \mathbb{Z} / p$, where the sum runs through all primes. Then $\mathscr{L}(G)^{\sim}=T_{Q}[G] \oplus \prod T_{P_{i}-Q}[G]$. Thus $c: G \rightarrow \mathscr{L}(G)^{\sim}$ is not an isomorphism whenever $\mathscr{Q}$ is an infinite partition of the set of primes. 
4.4 Example. Suppose $G$ is locally nilpotent and has torsion at only finitely many primes. Then $c: G \rightarrow \mathscr{L}(G)^{\sim}$ is an isomorphism for any choice of Q.

\section{MAPS FROM A PRODUCT OF TORSION GROUPS}

In this section we use local global arguments to calculate the set of homomorphisms from certain infinite products of torsion groups into a group $H$ for which the natural map $c: H \rightarrow \mathscr{L}(H)^{\sim}$ is an isomorphism. Our tool is the following interpretation of 1.8 in the category of groups.

5.1 Proposition. Suppose $H$ is a group such that the natural map $c: H \rightarrow$ $\mathscr{L}(H)^{\sim}$ is an isomorphism. For any group $G$ there is a natural bijection of morphism sets

$$
\mathscr{G}(G, H) \cong \mathscr{L} \mathscr{G}(\mathscr{L}(G), \mathscr{L}(H))
$$

5.2 Lemma. Let $\mathscr{Q}=\left\{Q ; P_{1}, P_{2}, \ldots\right\}$ be a relative partition of the set of primes. If $P_{\mu} \in \mathscr{Q}$, let $G_{\mu}$ be a $P_{\mu}$-local group. Set $K_{\mu}:=T_{P_{\mu}-Q}\left[G_{\mu}\right]$ and suppose the quotient $R_{\mu}:=G_{\mu} / K_{\mu}$ is $Q$-local. Then there is a short exact sequence

$$
\prod^{w} K_{\mu} \longmapsto \prod G_{\mu} \stackrel{\varepsilon}{\rightarrow} R
$$

in which $\varepsilon Q$-localizes.

Proof. By 3.6, $\prod G_{\mu}$ has $\prod^{w} K_{\mu}$ as a normal subgroup. Further, $\prod^{w} K_{\mu}=$ $T_{Q^{\prime}}\left[\Pi G_{\mu}\right]$. To see that $R$ is $Q$-local, let $n \in Q^{\prime}$. Then there are finitely many indices $\mu_{1}, \ldots, \mu_{s}$ such that $n$ is in the multiplicative closure of $\left(P_{\mu_{1}} \cup \cdots \cup\right.$ $\left.P_{\mu_{s}}\right)-Q$. By hypothesis, $n$th roots exist in $R_{\mu_{i}}, i=1, \ldots, s$, and in $G_{\mu}$ if $\mu \neq$ $\mu_{1}, \ldots, \mu_{s}$. Thus the $n$th power operation is onto in $\left(\prod G_{\mu}\right) /\left(K_{\mu_{1}} \times \cdots \times K_{\mu_{s}}\right)$ and hence in $R$.

To see that the $n$th power operation is $1-1$ on $R$, suppose ${\overline{\left(x_{\mu}\right)}}^{n}={\overline{\left(y_{\mu}\right)}}^{n}$ in $R$. Then $x_{\mu}^{n}=y_{\mu}^{n}$ for almost all $\mu$ and hence $x_{\mu}=y_{\mu}$ for almost all $\mu$. Thus $R$ is $Q$-local. The claim follows.

5.3 Theorem. Let $\mathscr{Q}=\left\{Q ; P_{1}, P_{2}, \ldots\right\}$ be a partition of the set of primes relative to $Q$, and let $H$ be a group in $\mathscr{G}_{Q}^{1}$ for which the natural map $c: H \rightarrow$ $\mathscr{L}(H)$ is an isomorphism. For each $\mu=1,2, \ldots$ let $G_{\mu}$ be a $\left(P_{\mu}-Q\right)$ torsion group. Then $\mathscr{G}\left(\prod G_{\mu}, H\right)$ corresponds bijectively to the set of all tuples $\left(v ; v_{1}, v_{2}, \ldots\right)$ satisfying

(i) $v \in \mathscr{G}\left(\prod G_{\mu} / \prod^{w} G_{\mu}, H\right)$ and $v_{\mu} \in \mathscr{G}\left(G_{\mu}, H\right)$;

(ii) $\operatorname{im}(v)$ commutes with $\operatorname{im}\left(v_{\mu}\right)$.

Proof. Set $G:=\prod G_{\mu}, K:=\prod^{w} G_{\mu}$, and $R:=G / K$. Invoking 5.1 we calculate $\mathscr{L} \mathscr{G}(\mathscr{L}(G), \mathscr{L}(H))$. By $5.2, \mathscr{L}(G)$ consists of the epimorphisms in the exact sequences

$$
G_{\mu} \longmapsto G / \prod_{\lambda \neq \mu}^{w} G_{\lambda}=L_{\mu} G \rightarrow R .
$$

The monomorphism in this sequence has a left inverse coming from the projection $G \rightarrow G_{\mu}$. Thus $L_{\mu} G \cong G_{\mu} \times R$ and $Q$-localization is the projection $\pi_{\mu}: G_{\mu} \times R \rightarrow R$. Thus a morphism $\mathscr{L}(G) \rightarrow \mathscr{L}(H)$ of $\mathscr{L}$-diagrams is given by a family of homomorphisms $\left(u ; u_{1}, u_{2}, \ldots\right)$ such that, for each $\mu$, the 
diagram below commutes:

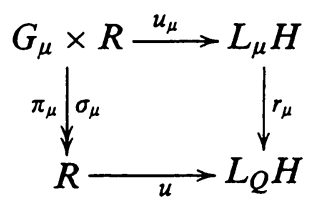

We show that the resulting sequences $\left(u ; u_{1}, \ldots\right)$ correspond bijectively to sequences $\left(v ; v_{1}, \ldots\right)$ as in the claim of the theorem. For each $\mu$ there is a cone $G_{\mu} \times R \rightarrow \mathscr{L}(H)$ given by $u \circ \pi_{\mu}: G_{\mu} \times R \rightarrow L_{Q} H$ and

$$
f_{\nu}^{\mu}: G_{\mu} \times R \rightarrow L_{\nu} H, \quad f_{\nu}^{\mu}:= \begin{cases}u_{\mu} & \text { if } \nu=\mu, \\ u_{\nu} \sigma_{\nu} \pi_{\mu} & \text { if } \nu \neq \mu .\end{cases}
$$

Thus $u_{\mu}$ lifts to a unique map $\widetilde{u_{\mu}}: G_{\mu} \times R \rightarrow H$ whose restriction to $G_{\mu}$ we denote by $v_{\mu}$ and whose restriction to $R$ we denote by $w_{\mu}$. It follows that

$$
\widetilde{u_{\mu}}\left(g_{\mu}, r\right)=v_{\mu}\left(g_{\mu}\right) \cdot w_{\mu}(r)=w_{\mu}(r) \cdot v_{\mu}\left(g_{\mu}\right) .
$$

Now $w_{\mu}: R \rightarrow H$ is the unique lift of the cone given by $u: R \rightarrow L_{Q} H$ together with $f_{\nu}^{\mu} \circ \sigma_{\mu}=u_{\nu} \circ \sigma_{\nu}: R \rightarrow L_{\nu} H$. So $w_{\mu}$ does not depend on the choice of $\mu$. So we may set $v:=w_{\mu}=w_{\nu}$. Hence a morphism $\mathscr{L}(G) \rightarrow \mathscr{L}(H)$ yields uniquely a sequence $\left(v ; v_{1}, v_{2}, \ldots\right)$ with the required properties.

Conversely, if such a sequence is given, localizing the product maps $v_{\mu} v: G_{\mu} \times$ $R \rightarrow H$ yields a morphism $\mathscr{L}(G) \rightarrow \mathscr{L}(H)$. Checking that these two processes are inverse to one another completes the proof.

\section{1-SIDED INVERSES FOR EPIMORPHISMS AND MONOMORPHISMS FROM LOCAL DATA}

6.1 Theorem. Let $\pi: G \rightarrow H$ be an epimorphism between groups for which the natural maps $G \rightarrow \mathscr{L}(G)^{\sim}$ and $H \rightarrow \mathscr{L}(H)^{\sim}$ are isomorphisms. Then $\pi$ has a section $\sigma: H \mapsto G$ if and only if $\mathscr{L}(\pi)$ has a section $\mathscr{L}(H) \rightarrow \mathscr{L}(G)$. Proof. Suppose $\underline{\sigma}: \mathscr{L}(H) \rightarrow \mathscr{L}(G)$ is a section of $\mathscr{L}(\pi)$ and consider the commutative diagram

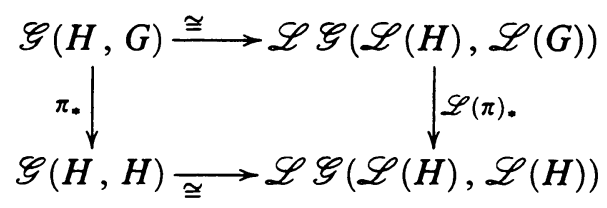

where the horizontal arrows are adjointness bijections; see 1.8. The section $\underline{\sigma}$ of $\mathscr{L}(\pi)$ corresponds uniquely to a homomorphism $\sigma: H \rightarrow G$. By commutativity of the diagram, $\pi \circ \sigma$ is the adjoint of $\mathscr{L}(\pi) \circ \underline{\sigma}=\operatorname{Id}_{\mathscr{L}(H)}$. Thus $\pi \circ \sigma=\mathrm{Id}_{H}$.

A similar result has been proven by Casacuberta and Hilton under the hypotheses that $G$ and $H$ are nilpotent and $\operatorname{ker}(\pi)$ has torsion at only finitely many primes; see [7]. 
6.2 Theorem. Let $i: N \rightarrow G$ be a monomorphism between groups for which the natural maps $N \rightarrow \mathscr{L}(N)^{\sim}$ and $G \rightarrow \mathscr{L}(G)^{\sim}$ are isomorphisms. Then $i$ has a left inverse if and only if $\mathscr{L}(i)$ has a left inverse.

Proof. Dualize the argument in 6.1 .

\section{THE $\mathscr{L}$-GENUS IN THE CATEGORY OF GROUPS}

Guided by the general framework of $\S 1$, we present the most basic facts governing the $\mathscr{L}$-genus of a group, where $\mathscr{L}$ is the diagram of localizing functors corresponding to a relative partition $\mathscr{Q}=\left\{Q ; P_{1}, P_{2}, \ldots\right\}$ of the set of all primes.

In this setting two groups $G$ and $H$ belong to the same $\mathscr{L}$-genus, if $\mathscr{L}(G) \cong$ $\mathscr{L}(H)$, i.e. if there are isomorphisms $u: L_{Q} G \rightarrow L_{Q} H$ and $u_{\mu}: L_{\mu} G \rightarrow L_{\mu} H$ such that, for each $\mu$, the diagram below commutes:

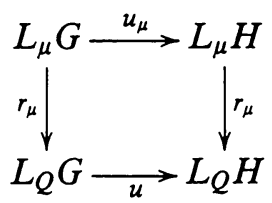

We also collect some basic properties of the class $\mathscr{L}_{*}$ of $\mathscr{L}$-genera together with its abelian semigroup structure defined by the coproduct "*" (usually called "free product"); see 1.5.

7.1 Remark. Let $\mathscr{G}_{*}$ denote the "semigroup", with respect to "free product", of isomorphism classes of groups. There is an obvious "semigroup" epimorphism $\mathscr{G}_{*} \rightarrow \mathscr{L}_{*}$. It has a nontrivial kernel, for there are groups, like the infinite alternating group, which become trivial if one inverts any prime. We call such groups $\mathscr{L}$-trivial. In the case where $\mathscr{L}$ is the discrete system $\left\{L_{2}, L_{3}, L_{5}, \ldots\right\}$, $L_{p}=$ localization at the prime $p$, this kernel has been considered in [3].

In order to suppress the influence of $\mathscr{L}$-trivial groups in genus considerations, one can restrict one's attention to groups in a suitable subcategory of $\mathscr{G}$. A good choice is the category $\mathscr{G}_{\mathbb{Q}}^{1}$ described in $\$ 3$.

In the proposition below we offer a simple criterion under which two groups $G, H$ in $\mathscr{G}_{\mathscr{Q}}^{1}$ satisfy $\mathscr{L}(G) \cong \mathscr{L}(H)$.

7.2 Proposition. Let $\underline{G}:=\left\{r_{\mu}: G_{\mu} \rightarrow G_{Q}\right\}$ be an $\mathscr{L}$-diagram in $\mathscr{G}_{\mathscr{Q}}^{1}$. Set $K_{\mu}:=\operatorname{ker}\left(r_{\mu}\right)$ and assume that the inclusion of $I:=\bigcap \operatorname{im}\left(r_{\mu}\right)$ into $G_{Q}$ induces isomorphisms $L_{\mu} I \rightarrow \operatorname{im}\left(r_{\mu}\right)$. Then the following hold.

(i) If an arbitrary group $\Gamma$ fits into the commutative diagram below, then $\mathscr{L}(\Gamma) \cong \underline{G}:$

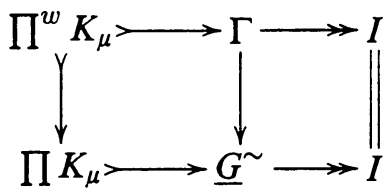

(ii) Any group $\Gamma$ in $\mathscr{G}_{\mathscr{Q}}^{1}$ which belongs to the $\mathscr{L}$-genus of $\underline{G}$ is an extension of $I$ by $\Pi^{w} K_{\mu}$.

Proof. (i) The adjoint of the given map $\Gamma \rightarrow \underline{G}^{\sim}$ is a morphism of diagrams $\mathscr{L}(\Gamma) \rightarrow \underline{G}$. We show first that $\Gamma \rightarrow I$ induces an isomorphism $L_{Q} \Gamma \stackrel{\cong}{\rightarrow} L_{Q} I \cong$ 
$G_{Q}$. Consider the commutative diagram below:

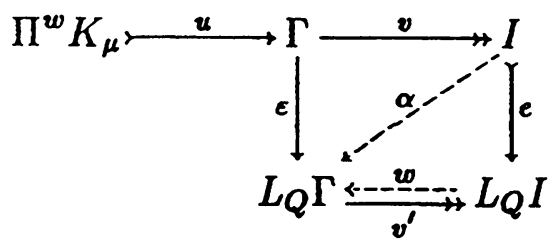

We know that $\operatorname{ker}(v)$ is contained in the kernel of the $Q$-localizing map $\varepsilon$. So $\varepsilon$ factors through $v$ via $\alpha$. The universal property of the $Q$-localizing map $e$ yields $w: L_{Q} I \rightarrow L_{Q} \Gamma$ with $\alpha=w e$. From the identity $v_{Q} \alpha v=v_{Q} \varepsilon=e v$ we see that $v_{Q} \alpha=e$. Now $v_{Q}$ and $w$ are inverse to each other, using the universal properties of $e$ and $\varepsilon$.

To see that $\mathscr{L}(\Gamma) \cong \underline{G}$ note first that $K_{\mu} \subset \operatorname{ker}\left(L_{\mu} v\right)$. On the other hand, $\operatorname{ker}(\varepsilon)=\operatorname{ker}(e v)$, and so $\operatorname{ker}\left(L_{\mu} v\right) \subset K_{\mu}$. Thus we get the commuting diagram below whose rows are exact:

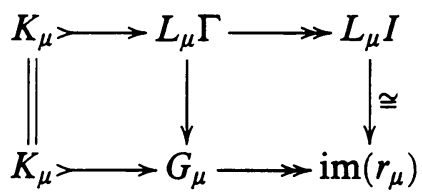

So $L_{\mu} \Gamma \rightarrow G_{\mu}$ is an isomorphism, implying that $\mathscr{L}(\Gamma) \cong \underline{G}$.

(ii) From 3.6 we see that $\operatorname{ker}\left(\Gamma \rightarrow L_{Q} \Gamma\right) \cong \Pi^{w} K_{\mu}$. Thus we have the short exact sequence

$$
\prod^{w} K_{\mu} \mapsto \Gamma \rightarrow J
$$

where $J$ is $Q^{\prime}$-torsion free and belongs to $\mathscr{G}_{\mathscr{Q}}^{1}$, using 2.2. By hypothesis we have the commutative diagram

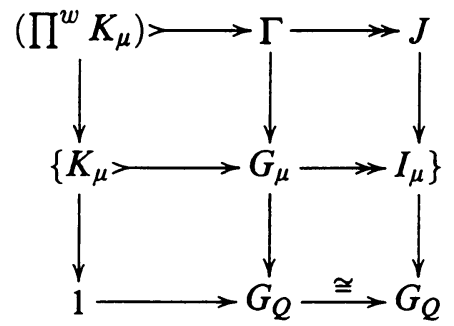

obtained by localizing the top row. By $4.1, J \cong \underline{I}^{\sim}$. The claim follows from the universal property of pull-backs, together with 2.4 .

Thus if $\Gamma \in \mathscr{G}_{\mathscr{Q}}^{1}$, then the groups $G \in \mathscr{G}_{\mathscr{Q}}^{1}$ which belong to the same $\mathscr{L}$-genus as $\Gamma$ are certain extensions of the $Q^{\prime}$-torsion free quotient of $\Gamma$ by $T_{Q^{\prime}}[\Gamma]$. If $G$ is abelian, it is possible to describe the isomorphism classes of abelian groups in the $\mathscr{L}$-genus of $G$ by means of homological algebra; see [16].

\section{ACKNOWLEDGMENT}

I learnt the argument used in the proof of 5.2 from Peter Symonds.

\section{REFERENCES}

1. J. F. Adams, Localisation and completion, Lecture Notes, Univ. of Chicago, 1975.

2. G. Baumslag, Roots and wreath products, Proc. Cambridge Philos. Soc. 56 (1960), 109-117. 
3. A. J. Berrick and C. Casacuberta, Groups and spaces with all localizations trivial, 1990 Barcelona Conference on Algebraic Topology, Lecture Notes in Math., vol. 1509, Springer, Berlin and New York, 1992, pp. 20-29.

4. N. Bourbaki, Theory of sets, Addison-Wesley, Reading, MA, and Don Mills, 1968.

5. A. K. Bousfield, The localization of spaces with respect to homology, Topology 14 (1975), 133-150.

6. L_ Localization and periodicity in unstable homotopy theory, preprint.

7. C. Casacuberta and P. J. Hilton, A note on extensions of nilpotent groups, Adams Memorial Symposium on Algebraic Topology, Vol. 1 (N. Ray and G. Walker, eds.), London Math. Soc. Lecture Notes Ser., vol. 175, Cambridge Univ. Press, London and New York, 1992, pp. 225-234.

8. C. Casacuberta and G. Peschke, Localizing with respect to self-maps of the circle, Trans. Amer. Math. Soc. 339 (1993), 117-140.

9. C. Casacuberta, G. Peschke, and M. Pfenniger, On orthogonal pairs in categories and localisation, Adams Memorial Symposium on Algebraic Topology (N. Ray and G. Walker, eds.), London Math. Soc. Lecture Notes Ser., vol. 175, Cambridge Univ. Press, London and New York, 1992, pp. 211-223.

10. P. J. Hilton, On direct limits of groups, Bol. Soc. Brasil. Mat. 2 (1970), 1-20.

11. __ Localization and cohomology of nilpotent groups, Math. Z. 132 (1973), 265-286.

12. __ On direct limits of nilpotent groups, Localization in Group Theory and Homotopy Theory, Lecture Notes in Math., vol. 418, Springer, Berlin and New York, 1974, pp. 68-77.

13. P. J. Hilton, G. Mislin, and J. Roitberg, Localization of nilpotent groups and spaces, NorthHolland, Amsterdam, 1975.

14. C. McGibbon, Clones of spaces and maps in homotopy theory, Comment. Math. Helv. 68 (1993), 263-277.

15. G. Peschke, Localizing groups with action, Publ. Mat. 33 (1989), 227-234.

16. G. Peschke and P. Symonds, Various local global principles for abelian groups, Publ. Mat. (to appear).

17. P. Ribenboim, Torsion et localisation de groupes arbitraires, Lecture Notes in Math., vol. 740, Springer-Verlag, Berlin, 1979, pp. 444-456.

18. Equations in groups, with special emphasis on localization and torsion I, Atti Accad. Naz. Lincei Mem. Cl. Sci. Fis. Mat. Natur. Ser. Ia (8) 19 (1987), 23-60.

19. Equations in groups, with special emphasis on localization and torsion II, Portugal. Math. 44 (1987), 417-445.

20. D. J. S. Robinson, $A$ course in the theory of groups, Graduate Texts in Math., vol. 80 , Springer-Verlag, Berlin and New York, 1982.

21. C. Wilkerson, Classification of spaces of the same n-type for all $n$, Proc. Amer. Math. Soc. 60 (1976), 279-285.

Department of Mathematical Sciences, University of Alberta, Edmonton, Canada T6G 2G1

E-mail address: gepe@jazz.math.ualberta.ca 